\title{
Las teorías subalternas de las Relaciones Internacionales y los mundos alternativos posibles
}

The subaltern theories of International Relations and the possible alternative worlds

\author{
Mario Iván Urueña-Sánchez*
}

Resumen: El objetivo de este artículo es el de visualizar la posibilidad de mundos alternativos al actual a partir del análisis de la integridad teórica interna de las teorías subalternas de las Relaciones Internacionales (Neogramscismo, Teoría Crítica, Posestructuralismo, Feminismo y Poscolonialismo). Para dar cumplimento a dicho objetivo se realizará una investigación de índole documental. La metodología escogida para tal efecto es un análisis documental que aspira a diseccionar los argumentos principales, los conceptos clave y las valoraciones realizadas por exponentes emblemáticos de las teorías subalternas. La principal conclusión a la que llega este artículo es que a pesar de unas inquietudes comunes sobre la manera de superar las estructuras ideacionales de dominación, las teorías subalternas enfrentan obstáculos aparentemente insalvables que pueden poder en riesgo la posibilidad de subvertir el orden establecido.

Palabras clave: teorías subalternas, mundos alternativos, integridad teórica interna, contrahegemonía.

\begin{abstract}
The objective of this article is to visualize the possibility of alternative worlds to the current one from the analysis of the internal theoretical integrity of the subaltern theories of International Relations (Neogramscism, Critical Theory, Post-structuralism, Feminism and Post-colonialism). To fulfill this objective, a documentary investigation will be carried out. The methodology chosen for this purpose is a documentary analysis that aims to dissect the main arguments, key concepts and assessments made by emblematic exponents of subaltern theories. The main conclusion reached by this article is that despite common concerns about how to overcome the ideational structures of domination, subaltern theories face seemingly insurmountable obstacles that may jeopardize the possibility of subverting the established order.
\end{abstract}

\footnotetext{
*Nacido en Colombia. Doctor en Derecho de la Universidad del Rosario (Colombia). Estudios de doctorado en Ciencia Política, Universidad de Quebec en Montreal (Canadá). Magíster en Geopolítica y Seguridad Global, Universidad de Roma "La Sapienza” (Italia). Politólogo, Universidad Nacional de Colombia. Profesor Facultad de Jurisprudencia, Universidad del Rosario. Correo: mario.uruena@urosario.edu.co.
} 
Keywords: subordinate theories, alternative worlds, internal theoretical integrity, counterhegemony.

Recibido: 20 agosto 2019 Aceptado: 19 octubre 2019

\section{Introducción}

¿Cómo el entendimiento de las realidades internacionales puede coadyuvar a la construcción de mundos alternativos posibles? Esta pregunta de investigación se inspira en la apatía percibida en los discursos dominantes de las Relaciones Internacionales con respecto a las inquietudes éticas, morales o incluso ideológicas que puede generar la puesta en práctica de la teorización en este campo de estudio.

Esta preocupación va más allá de un diagnóstico por la normatividad de las teorías de las Relaciones internacionales y procura la viabilidad que desde estas puede existir para la concepción de mundos alternativos posibles. El pesimismo inherente al Realismo y la progresividad reformista de los liberalismos y el Constructivismo racionalista crean una sensación de desaliento bien sea porque invitan a conformarse con el estado actual de las cosas o a esperar cambios menores solo perceptibles en el largo plazo.

El objetivo de este artículo es entonces el de visualizar la posibilidad de mundos alternativos al actual a partir del análisis de la integridad teórica interna de las teorías subalternas de las Relaciones Internacionales (Neogramscismo, Teoría crítica, Posestructuralismo, Feminismo y Poscolonialismo). El plantear un trabajo sobre las teorías subalternas representa un desafío mayúsculo por cuanto obliga a desaprender la construcción del conocimiento positivista. El abandono de la idea clásica de causalidad obliga a problematizar la relación entre ontología y epistemología y analizar las repercusiones de esta interacción sobre la normatividad.

Para dar alcance a este objetivo se ha fijado un foco de investigación consistente en las cinco teorías subalternas arriba mencionadas con la intención de ponerlas a dialogar en búsqueda de confluencias, discrepancias y especialmente acerca de su propuesta de transformación social. La metodología escogida para tal efecto es un análisis documental que aspira a diseccionar los argumentos principales, los conceptos clave y las valoraciones realizadas por exponentes emblemáticos de las teorías subalternas.

Este artículo se encuentra dividido en tres partes. En la primera parte, se realiza un encuadramiento de las teorías subalternas en el programa de investigación de las Relaciones Internacionales. Este encuadramiento se delimita a partir de los llamados grandes debates disciplinares para perfilar unos elementos de definición, identificar los rastros comunes de las teorías subalternas y brindar unos elementos de definición más precisos. La segunda parte resalta el modo en el que ontología y epistemología se interrelacionan en la construcción teórica de las perspectivas subalternas. Su ruptura con el positivismo, los ejes articuladores derivados de esta interrelación y las discusiones que entre las propias teorías 
subalternas suscitan estas preocupaciones dan desarrollo a esta segunda parte. En la tercera parte, la inquisición por la normatividad toma protagonismo con miras a contrastar las propuestas de cada teoría subalterna respecto a las líneas de acción que entrevean mundos futuros diferentes a los prefijados. Al poner en relieve su explicitud normativa, el foco de los valores promovidos y la agenda de cambio social e internacional se recrean las prácticas por seguir en el porvenir.

\section{El encuadramiento de las teorías subalternas: ¿Cuál es su lugar en los debates de las Relaciones Internacionales?}

Al hablar de teorías subalternas en el campo de investigación de las Relaciones Internacionales, es evidente que estas hacen parte de un espectro ajeno a las corrientes teóricas principales de este campo. Por ello, es pertinente realizar un encuadramiento de dichas teorías a partir de i) los denominados grandes debates de la disciplina de las Relaciones Internacionales, ii) las tipologías que de estos debates se encuentran disponibles para delimitar a las teorías subalternas, para iii) vislumbrar las orientaciones epistemológicas que facultan el entendimiento del universo de las teorías subalternas.

\section{Los grandes debates de las Relaciones Internacionales.}

La discusión con respecto a los denominados grandes debates de las Relaciones Internacionales dista de estar exenta de la polémica acerca del número de estos. Esta discusión ha ido a los extremos de considerar por un lado que no han existido grandes debates en la disciplina mientras por el otro consideran que han existido cinco o más grandes debates. La imposibilidad de los marcos analíticos puestos en discusión para llevar a cabo una construcción colectiva debido a la tendencia de estos a ignorarse los unos a los otros ha conducido a algunos autores como Steve Smith a restarle valor al uso del término "debates" para calificar lo ocurrido en el programa de investigación de las Relaciones Internacionales. En el extremo opuesto, el propio Steve Smith ha desestimado también a aquellos teóricos que señalan sus aportes como constitutivos de la emergencia de un quinto gran debate disciplinar. La entrada en escena del Realismo científico como orientación epistemológica llamada a revolucionar el campo de estudio carece de sentido y puede terminar siendo igual de inadmisible que los, para él, cuatro debates anteriores. ${ }^{2}$

La anterior reflexión dista de ser mayoritaria entre los tratadistas de las Relaciones Internacionales. Entre ellos, el "debate sobre los grandes debates" se centra entre quienes defienden la existencia de tres debates y quienes apuntan a determinar su número en cuatro. La publicación en 1988 del artículo de Robert Keohane titulado International Institutions: Two Approaches en el cual este autor estadounidense contrapone a las teorías racionalistas y a las reflexivistas. Para Keohane, si bien las segundas aciertan en su crítica a las primeras para

\footnotetext{
2 Steve Smith, "Six Wishes for a More Relevant Discipline of International Relations", Christian Reus-Smit y Duncan Snidal (eds.), The Oxford Handbook of International Relations, Oxford University Press, Oxford, 2008, 725-732.
} 
urgirlas por una contextualización histórica, puesto que los análisis racionalistas tienen límites para explicar instituciones específicas, su catalogación como teorías es menos claro. Ello por cuanto las teorías reflexivistas han preferido acusar los defectos y omisiones de su contraparte racionalista en vez de desarrollar un programa de investigación coherente por ellas mismas. ${ }^{3}$

Aunque Keohane no encuadra este debate en un orden específico, su planteamiento sirvió de inspiración para que otros autores lo ubicaran en un contexto más amplio. Por un lado, autores como Yosef Lapid hacen una leve modificación a la propuesta de Keohane al cambiar la dicotomía racionalista/reflexivista por la positivista/postpositivista para postular entonces la existencia de un tercer gran debate en las Relaciones Internacionales. Luego del debate dogmático (idealistas vs. realistas) del periodo entreguerras y del debate metodológico (tradicionalistas vs. cientificistas) producido después de la revolución behaviorista, la confrontación entre positivistas y postpositivistas marcaría el paso a una tercera versión de antagonismos disciplinares. Una vez establecido este tercer debate, Lapid arriba a una conclusión similar a la de Keohane: las visiones postpositivistas pueden abrir varios caminos prometedores para investigaciones futuras como puede terminar llevando de la misma manera a una cantidad similar de callejones sin salida. ${ }^{4}$

Por el otro lado, Ole Waever hace una relectura acerca de la cantidad de debates disciplinares. Según él, la sabiduría instituida establece tres debates de las Relaciones Internacionales, pero el precedente de un debate interparadigmático en la década de 1970 entre realistas, transnacionalistas y marxistas reconfiguró la organización de los debates. ${ }^{5}$ La crítica liberal (transnacionalistas) a los realistas se concentró en cuestiones ontológicas e ignoró los problemas epistemológicos, puesto que en este frente primaba el acuerdo entre ambos paradigmas. Esta coincidencia impulsó al marxismo a poner en cuestionamiento a las Relaciones Internacionales en términos muy diferentes a los propuestos por el debate Liberalismo/Realismo. ${ }^{6}$ Yendo más allá de la inclusión del debate interparadigmático, Waever adhiere otro debate a lo previamente determinado: la síntesis neo-neo. Para él:

Durante la década de 1980, el realismo se convirtió en neorrealismo y el liberalismo en institucionalismo neoliberal. Ambos se sometieron a una redefinición autolimitada hacia un minimalismo teórico y anti-metafísico y, por lo tanto, se hicieron cada vez más compatibles. Una síntesis neo-neo dominante se convirtió en el programa de investigación de los años 80.7

\footnotetext{
${ }^{3}$ Robert Keohane, “International Institutions: Two Approaches”, International Studies Quaterly 32:4, diciembre 1988, 379-396.

4 Yosef Lapid, "The Third Debate: On the Prospects of International Theory in a Post-Positivist Era", International Studies Quaterly 33:3, septiembre 1989, 235-254.

5 Ole Waever, "The rise and fall of the inter-paradigm debate", Steve Smith, Ken Booth y Marysia Zalewski (eds.), International theory: Positivism \& beyond, Cambridge University Press, Cambridge, 1996, 149-185.

6 Alex Macleod, "Émergence d'un paradigme hégémonique", Alex Macleod y Dan O’Meara, Théories des relations internationales : contestations et résistances, Athéna éditions, Outremont, 2010, 19-35.

7 Waever, op.cit, 149-185.
} 
Esta compatibilidad entre visiones liberales y realistas condujeron a Waever a observar una variante empirista del debate entre racionalistas y reflexivistas. Con todo esto en mente, el siguiente diagrama ilustra la visión del profesor danés sobre el tercer y cuarto debate del campo de las Relaciones Internacionales.

\begin{tabular}{|c|c|c|c|}
\hline & $\begin{array}{c}\text { 3er debate (debate } \\
\text { inter-paradigmático) }\end{array}$ & $\begin{array}{c}\text { Debate 4a } \\
\text { (reflexivistas- } \\
\text { racionalistas) }\end{array}$ & $\begin{array}{c}\text { Debate 4b (ganancias } \\
\text { absolutas-relativas) }\end{array}$ \\
\hline $\begin{array}{c}\text { Forma de relación } \\
\text { entre quienes } \\
\text { debaten }\end{array}$ & Inconmensurabilidad & Guerra & $\begin{array}{c}\text { Diferencias al } \\
\text { interior de un } \\
\text { programa de } \\
\text { investigación }\end{array}$ \\
\hline $\begin{array}{c}\text { Tema (o sustancia) } \\
\text { del desacuerdo }\end{array}$ & Visión del mundo & Filosofía & $\begin{array}{c}\text { Cuestiones empíricas } \\
\text { por organizar }\end{array}$ \\
\hline Combatientes & Los tres paradigmas & $\begin{array}{c}\text { Síntesis neo-neo } \\
\text { contra los } \\
\text { posmodernistas }\end{array}$ & $\begin{array}{c}\text { Neorrealistas contra } \\
\text { los institucionalistas } \\
\text { neoliberales }\end{array}$ \\
\hline
\end{tabular}

Tomado de Waever, op. cit, p. 167.

La ascendencia de la tesis de Waever entre múltiples autores de las Relaciones Internacionales no ha estado exenta de reparos. A pesar de resultar un debate enriquecedor por involucrar el pluralismo en la disciplina e ir más allá de la visión realista dominante hasta ese momento, la historia oficial de este campo de estudio excluye al debate interparadigmático por juzgarlo fragmentador de la disciplina y por no haber durado el suficiente tiempo para hacerse acreedor al título de "tercer debate" .8 Es por lo tanto que hay una visión dominante entre los estudiosos de la materia que limitan a tres el número de debates reconocidos.

\section{Las tipologías disponibles de las teorías de las Relaciones Internacionales.}

A propósito de la discusión sobre los grandes debates de las Relaciones Internacionales, algunos autores de la disciplina propusieron ofrecer una serie de categorizaciones para distinguirlas. Como se mencionó, Robert Keohane y Yosef Lapid ofrecen respectivamente las categorizaciones racionalismo/reflexivismo y positivismo/postpositivismo. Aunado a estos binomios, se mostrarán a continuación otras categorizaciones preconizadas por autores como Martin Hollis y Steve Smith y por Robert Cox. Para los dos primeros autores, se mirará la oposición entre teorías explicativas y teorías del entendimiento mientras que en el caso del último autor se hará el ejercicio respectivo con las teorías de resolución de problemas y las teorías críticas.

En primer lugar, las teorías racionalistas son aquellas que conciernen a un enfoque epistemológico que pretende que el conocimiento debe basarse en primera medida en la

${ }^{8}$ Macleod, op. cit, 19-35. 
inteligencia y en el razonamiento a priori. ${ }^{9}$ Las teorías reflexivistas pueden ser definidas como:

[...] una reflexión en el proceso de teorización. Más específicamente, se puede entender que la reflexividad en términos de la teoría de las Relaciones Internacionales conlleva tres elementos centrales: 1) Autoconciencia con respecto a las premisas subyacentes; 2) el reconocimiento de la dimensión político-normativa inherente de los paradigmas y la tradición científica normal que sostienen; 3) la afirmación de que los juicios razonados sobre los méritos de los paradigmas en conflicto son posibles en ausencia de un lenguaje de observación neutral. ${ }^{10}$

En segundo lugar, las teorías positivistas asumen presupuestos relativos a la unidad de las ciencias (naturalismo), la distinción entre hechos y valores, a que tanto el mundo social como el natural comportan regularidades que pueden ser descubiertas a través de las teorías y que la verdad acerca de una proposición debe pasar por la corroboración de los hechos. Las teorías postpositivistas de su parte no piensan en la posibilidad de distinguir entre hechos y valores, tampoco a la visión de un mundo restringido a Estados que interactúan en un sistema internacional anárquico, además de ser teorías totalmente normativas. ${ }^{11}$

En tercer lugar, las teorías explicativas se identifican por ser una narración del mundo exterior de las Relaciones Internacionales la cual busca encausar el funcionamiento del reino de la naturaleza dentro del cual se circunscribe la comunidad humana. Las teorías del entendimiento toman otro rumbo al preocuparse de una narrativa del mundo interior, en el cual se intenta dar interpretación al significado de los eventos, en un sentido distinto al de las leyes de la naturaleza. ${ }^{12}$ En cuarto lugar, las teorías de resolución de problemas tienen como propósito "guiar a resolver problemas planteados en términos de una perspectiva particular la cual sirve de punto de partida". ${ }^{13}$ Las teorías críticas tienen de su parte el propósito de "llegar a tener plena conciencia de la perspectiva desde la cual emerge la teorización y su relación con otras perspectivas (para lograr una perspectiva de perspectivas), y para abrir la posibilidad de escoger una perspectiva diferente y válida desde la cual la problemática termina siendo la de crear un mundo alternativo" ${ }^{14}$

\footnotetext{
9 Ibid, p. 21.

10 Mark Neufeld, "Reflexivism”, Claire Turenne Sjölander y Wayne S. Cox (dirs,), Beyond Positivism: Critical International Relations Theory, Boulder, Lynne Rienner Publications, 1994. 13-45.

11 Macleod, op. cit, p. 27-29.

12 Martin Hollis y Steve Smith, Explaining and Understanding International Relations, Oxford, Clarendon Press, 1990, p. 1.

13 Traducción libre del autor del inglés. El texto original reza "Problem-solving (P-S) theory purpose: to be guide to help solve the problems posed within the terms of the particular perspective which was the point of departure". Robert Cox y Timothy Sinclair, Approaches to World Order, Cambridge, Cambridge University Press, 1996, p. 88.

14 Traducción libre del autor. El texto original reza "Critical (C) theory purpose: to become clearly aware of the perspective which gives rise to theorizing, and its relation to other perspectives (to achieve a perspective on perspectives); and to open up the possibility of choosing a different valid perspective from which the
} 
Una vez reconstruidas estas categorizaciones de las teorías de las Relaciones Internacionales, se identifican trazos en común entre los tipos de categorías. De un lado, las teorías positivistas, racionalistas, explicativas y de resolución de problemas coinciden, con matices, en la búsqueda de la verdad mediante la experiencia, los sentidos y la razón humana para lo cual se valen de método científico y el rastreo de regularidades y causalidades. Del otro lado, las teorías postpositivistas, reflexivistas, del entendimiento y críticas se encuentran en elementos como la introspección de los valores del sujeto, en la imposibilidad de escindir hechos de valores, en la necesidad de contextualizar históricamente, en el pluralismo metodológico y en la normatividad explícita y transformadora que debe orientar a una teoría. Entre los dos grupos mencionados, las teorías subalternas hallan su razón de ser y se identifican claramente con el segundo. Ello a pesar de que su universo de definición contemple una delimitación particular.

\section{Las teorías subalternas: criterios de delimitación conceptuales.}

Como eje central de este artículo, las llamadas teorías subalternas comparten muchos de los preceptos defendidos por el segundo grupo de teorías arriba mencionadas. Teorías que, como lo señala Ole Waever, se encuentran en guerra con las ópticas dominantes de las Relaciones Internacionales como lo son el debate entre neorrealistas e institucionalistas liberales. Particularmente con los Neorrealistas o Realistas estructurales, las teorías subalternas son fuertemente críticas debido a que estos primeros edificaron el discurso dominante de la política exterior de las potencias hegemónicas durante la segunda mitad de la Guerra Fría. Los principales señalamientos de las teorías subalternas al Neorrealismo apuntan en un primer momento a su imposibilidad de explicar el cambio en el sistema internacional, a su Estado-centrismo y a su elitismo, tanto internacional como doméstico.

En primera instancia, una crítica constante al Neorrealismo es su debilidad para dar cuenta de la posibilidad que tiene el sistema internacional para sufrir trasformaciones. Para los autores de esta postura, la visión del sistema internacional y de su estructura son estáticos. Poco abiertos a renovarse o a cambiar. Su principal exponente, Kenneth Waltz fracasa en brindar una formulación sobre la génesis de la estructura de este sistema, limitándose a exponer una imagen descontextualizada y simplificada de la misma. Por ello, el Neorrealismo, más que una teoría es un modelo cuyo panorama de la sociedad lleva inserto en sí una lógica reproductiva, no transformadora. ${ }^{15}$ Otro exponente neorrealista, Robert Gilpin, ilustra esta premisa con suficiencia al afirmar en su principal obra "el supuesto fundamental de este estudio ha sido que la naturaleza de las relaciones internacionales no ha cambiado fundamentalmente a través de los últimos milenios". ${ }^{16}$

En segunda instancia, el Neorrealismo restringe la diversidad de actores del sistema internacional a los Estados y al sistema mismo. Para ellos, los demás actores están subordinados a la correlación de fuerzas entre los Estados y a los constreñimientos

problematic becomes one of creating an alternative world". Robert Cox y Timothy Sinclair, Approaches to World Order, Cambridge, Cambridge University Press, 1996, p. 88.

15 Robert Keohane, Neorealism and Its Critics, Nueva York, Columbia University Press, 1986, p. 159.

16 Robert Gilpin, War and change in world politics, Cambridge, Cambridge University Press, 1981, p. 281. 
sistémicos al comportamiento de estos. Si bien Waltz se aleja del Realismo de Hans Morgenthau al acusarlo de ser una teoría reduccionista en tanto explica los resultados de la política internacional solo a partir de la combinación de elementos al nivel nacional o subnacional, 17 este autor conserva una suerte de estatismo utilitarista como el núcleo de su programa de investigación. ${ }^{18}$

En tercera instancia, al ser una teoría sistémica, el Neorrealismo privilegió en sus análisis a las unidades cuyo peso específico tuviera capacidad de afectación del sistema internacional. Al tomar una decisión en tal dirección, los neorrealistas se decantaron por una epistemología de "arriba hacia abajo" de las Relaciones Internacionales cuya observación está focalizada en las unidades con mayores capacidades materiales del sistema. La participación de unidades de mediana o baja capacidad es prácticamente desestimada por los neorrealistas. Como factor adicional, esta preponderancia de unidades parte en primera medida por las relaciones de orden político militar que tengan estas unidades para equilibrar su poderío con el de otras facciones. ${ }^{19}$

En cuarta instancia, la predilección neorrealista por las unidades estatales más poderosas del sistema ni siquiera conlleva a una preocupación por la manera en que se organizan estas. La idea del actor unitario presume un comportamiento de las unidades homogéneo y predecible. Sin embargo, este comportamiento termina por recaer finalmente sobre las élites políticas y militares de estos Estados. ${ }^{20}$ Los tomadores de decisión en estos frentes parecen actuar en acuerdo a esta óptica como entidades aisladas del entorno social y las correlaciones de fuerza internas, siempre bajo los imperativos del interés nacional y la supervivencia. Sin más fuerzas que les hagan contrapeso a estos imperativos.

Al reclamarse como parte de las críticas radicales al Neorrealismo, las teorías subalternas deben ser definidas en un inicio en oposición a los anteriores puntos. Por lo tanto, el término "subalternas" hace alusión a problematizar el origen y desenlace del devenir del sistema internacional con vista a la posibilidad de que este sea transformado, a abandonar el Estadocentrismo y contemplar dinámicas internacionales que comprometen a una gran diversidad de actores y de formas de interacción entre ellos, a primar la visión "desde abajo" de las Relaciones Internacionales, sea para observar las regiones periféricas como para poner en foco la visión desde los excluidos y desposeídos del orbe. Este ejercicio de problematización partirá entonces de adelantar una discusión que atraviese por igual las dimensiones ontológicas, epistemológicas y especialmente normativas de la construcción teórica y cuyos pilares fundamentales serán abordados en las secciones siguientes.

\footnotetext{
17 Kenneth Waltz, Theory of International Politics, Nueva York, Columbia University Press, 1979, p. 34.

18 Richard Ashley, "The Poverty of Neorealism", Robert O. Keohane (ed.), Neorealism and Its Critics, op. cit, 1986, p. 276.

19 Ibid, pp. 260-261.

${ }^{20}$ Keith Tooper, “The Theory of International Politics? An Analysis of Neorealist Theory”, Human Studies 21: 2, abril de 1998, 157-186.
} 


\section{Ontología y epistemología en las teorías subalternas de las Relaciones Internacionales: dos caras de una misma moneda}

Al haber establecido la categorización de las teorías subalternas a partir de directrices ontológicas, epistemológicas y normativas, se hace necesario entablar unas líneas de análisis para entender la integridad teórica interna de este enfoque. En este apartado del artículo, se tomará la interacción entre las dos primeras en las teorías subalternas para: i) valorar sus puntos de ruptura con el positivismo, ii) remarcar al historicismo y al interpretivismo como ejes de la articulación entre estas teorías y iii) definir unas líneas de discusión al interior de las propias teorías subalternas.

\section{Las teorías subalternas de cara a las visiones positivistas de las Relaciones Internacionales.}

En lo concerniente a la relación entre ontología y epistemología, huelga anotar una primera distinción entre las teorías positivistas y las teorías subalternas de las Relaciones Internacionales. Mientras en las primeras, ontología y epistemología aparecen como dos dimensiones separadas, en las teorías subalternas, ellas se plantean como dimensiones inescindibles. Como dos caras de una misma moneda. A continuación, se desarrollarán algunas consideraciones acerca del binomio ontología/epistemología en ambas visiones teóricas.

En primer lugar, el positivismo como orientación epistemológica parte de tres principios básicos. Estos son: i) La creencia en la verdad como correspondencia entre la teoría y los hechos, ii) la unidad metodológica de la ciencia y iii) el conocimiento científico cuya naturaleza es libre de valores. Estos principios se basan a su vez en tres supuestos básicos: i) la separación entre sujeto y objeto, ii) el naturalismo y iii) la separación de hecho y valor. ${ }^{21}$

En el campo de las Relaciones Internacionales, la ontología de las teorías positivistas parte de una visión preeminentemente materialista de la realidad internacional, en contraste a las subalternas, las cuales se inclinan por privilegiar una ontología ideacionalista. Dentro de la primera óptica, la síntesis neo-neo pone de presente el marcado materialismo que une a las vertientes positivistas. La disquisición sobre si es el armamento (Neorrealistas) o la riqueza (Institucionalismo neoliberal) la prioridad para la seguridad de los Estados encuadra a esta síntesis en una preferencia por aquello que se puede observar, cuantificar y medir. Cabe aclarar que el peso de las capacidades materiales está matizado en el Institucionalismo neoliberal debido a que autores como Keohane rescatan la importancia de las cuestiones intersubjetivas para motivar a los Estados a cooperar. ${ }^{22}$

Esta primacía del materialismo ontológico en el positivismo es relativizada en el plano del positivismo social. En este se puede distinguir entre un behaviorismo estricto, en el cual la conciencia humana se reduce a la caza de regularidades para formular leyes, y un

${ }^{21}$ Mark Neufeld, The restructuring of International Relations theory, Cambridge, Cambridge University press, 2002, p. 21.

22 Keohane, op. cit, 1988. 
behaviorismo orientado al significado cuyo espectro contempla significados subjetivos. Para ambas vertientes de positivismo social la verstehen weberiana es útil en función del descubrimiento, ya que en su concepto de "acción social" los significados subjetivos pueden ser asimilados sin poner en riesgo la noción de "adecuación causal" ${ }^{23}$

En sentido opuesto, para las teorías subalternas elementos como el poder, la hegemonía o las estructuras no pueden ser definidos únicamente en términos materiales. Aunque teorías como el Neogramscismo no desestiman los aspectos materiales, ellos afirman que ese poder o esa hegemonía tiene su contraparte en el mundo de las ideas. Allí, la ideología sirve de apoyo a la configuración de las correlaciones de poder al movilizar opiniones favorables a este. La Hegemonía es una fuerza mutable en tiempo que depende tanto de la variaciones materiales e ideacionales del cambio de las relaciones de poder en el tiempo. ${ }^{24}$ En el Posestructuralismo, la estructura está basada en discursos que juegan un papel preponderante en la construcción subjetividades, objetividades y conductas de los actores. ${ }^{25}$ Para ellos, los emisores de esos discursos usan y son usados por el lenguaje, la retórica, las narrativas y la gramática, los cuales conforman unas prácticas de significación indeterminada y ambigua. ${ }^{26}$

En segundo lugar, la distinción entre perspectivas explicativas y constitutivas marcan un contraste al valorar la relación entre hechos y valores. En las primeras, preconizadas por las teorías positivistas, la pretensión de rastrear relaciones causales entre variables adquiere sentido en la explicación, el análisis e incluso en la predicción de los fenómenos internacionales. Para Waltz explicar es "decir por qué el rango de resultados esperados cae dentro de ciertos límites; decir por qué los patrones de comportamiento se repiten; para decir por qué los eventos se respetan a sí mismos, incluidos los eventos que a ninguno de los pocos actores les puede gustar" ${ }^{27}$ Es en el rastreo de esas regularidades que las teorías logran tener un poder predictivo general para visualizar escenarios futuros. Las teorías constitutivas se interesan más en definir el "cómo" que el "por qué" del funcionamiento y de emergencia de las cosas a través de la interpretación del mundo interior del objeto de análisis. Ellas priorizan así la exploración de los procesos en vez de las causalidades de los fenómenos. ${ }^{28}$

El aparente universalismo de las teorías explicativas desvela desde las teorías constitutivas una visión totalizante que no deja de desprenderse de un enfoque particular. Este afán de generalización es resumido en la noción del "punto arquimédico". Este punto arquimédico presume de un observador que se encuentra más allá del orbe y de la historia, cosa que le brinda una visión general y objetiva de aquello que estudia. Una visión que no

\footnotetext{
${ }^{23}$ Marc Neufeld, “Interpretation and the 'Science' od International Relations", Review of International Studies 19:1, enero de 1993, 39-61.

24 Cox y Sinclair, op. cit, p. 364.

${ }^{25}$ Ashley, op. cit.

26 James Der Derian, “The (S)pace of International Relations: Simulation, Surveillance and Speed”, International Studies Quaterly 34:3, septiembre de 1990, 295-310.

27 Traducción libre del autor. El texto original reza "to say why the range of expected outcomes falls within certain limits; to say why patterns of behavior recur; to say why events respect themselves., including events that none of few of the actors may like". Waltz, op. cit, p. 72.

${ }^{28}$ Hollis y Smith, op. cit, pp.45-91.
} 
deja de proceder de un punto particular. Entre las teorías subalternas, los neogramscianos reafirman esta intención al apuntar a que "las teorías de resolución de problemas asimilan situaciones particulares a reglas generales, proporcionando el tipo de método programado para tratar con ellos". 29

Para los poscolonialistas, esta visión totalizante de la disciplina de las Relaciones Internacionales, nacida en Europa y dominada actualmente por la influencia académica estadounidense, cuenta con unas corrientes principales focalizadas en el discurso de los poderosos del mundo. Estos discursos solo ofrecen una "visión del mundo" vista desde occidente, la cual falla en involucrar las preocupaciones y perspectivas surgidas de los países y poblaciones más pobres. ${ }^{30}$ En varios feminismos, el concepto de deconstrucción significa la interpretación documental sobre la base del sesgo androcéntrico y de la codificación cultural de los hombres como los "conocedores", excluyendo a las mujeres como agentes de cualquier posible cambio social. ${ }^{31}$ Es claro entonces que el punto arquimédico que ha atravesado a las teorías positivistas en las Relaciones Internacionales ha estado marcado por el particularismo, el elitismo y el machismo.

Derivado de esta noción de punto arquimédico aparece la "ansiedad cartesiana", otro fundamento de la racionalidad positivista que Richard J. Bernstein describió como "la proposición modernista que afirma que o bien tenemos algún tipo de 'fundamento' definitivo para nuestro conocimiento o estamos sumidos en el vacío de lo relativo, lo irracional, lo arbitrario, lo nihilista" ${ }^{32}$ La ansiedad cartesiana demanda en consecuencia una separación entre el mundo exterior y el sujeto que observa.

Las teorías constitutivas rechazan por igual el punto arquimédico y la ansiedad cartesiana. Los poscolonialistas se enfocan en el "Sur" y en los pueblos marginalizados con el objeto de inquirir por la importancia de las especificidades y de las experiencias "situadas diferencialmente". Se intenta así dar cuenta de las relaciones coloniales para entender por igual a los (ex)colonizadores y a los (des)colonizados. ${ }^{33}$ Los feminismos anteponen al concepto de deconstrucción el de reconstrucción. Este último concepto marca el cambio a:

Recuperarnos [a las mujeres] hasta examinar críticamente el mundo desde la perspectiva de esta recuperación [...] Un movimiento del margen al centro. No solo buscando acceder y participar dentro de (sino desde los márgenes de) paradigmas androcéntricos, la reconstrucción feminista explora las implicaciones teóricas de revelar sesgos sistémicos masculinos y agregar sistemáticamente mujeres. ${ }^{34}$

\footnotetext{
${ }^{29}$ Cox y Sinclair, op. cit, p. 53.

30 Rita Abrahamsen, "Postcolonialism", Martin Griffiths (ed.), International Relations Theory for the TwentyFirst Centruy. An Introduction, Nueva York y Londres, Routlegde, 2007, 111-122.

31 Christine Sylvester, "The constributions of feminist theory to international relations", Steve Smith, Ken Booth y Marysia Zalewski (eds.), International theory: Positivism \& beyond, Cambridge University Press, Cambridge, 1996, 254-278.

32 Traducción libre del autor. El texto original reza 'the modernist proposition which asserts that either we have some sort of ultimate 'foundation' for our knowledge or we are plunged into the void of the relative, the irrational, the arbitrary, the nihilistic'. Citado en Neufeld, op. cit, 1995, p. 62.

33 Abrahamsen, op. cit, p. 112.

34 Sylvester, op. cit, p. 258.
} 
Con respecto a la ansiedad cartesiana, esta separación de hechos y valores es una quimera para los subalternos puesto que para ellos el verdadero conocimiento de las cosas depende del pensamiento para ser concebido y no a la inversa. Esta indivisibilidad entre hechos y valores, o entre objeto y pensamiento, fue preconizada por Immanuel Kant bajo el rótulo del "giro copernicano". 35

En esta distinción teórica las teorías subalternas se identifican en mayor medida como teorías constitutivas. Teorías con vocación de interpretación y en las cuales hechos y valores son inseparables. Para ellas, la intención de generalización y objetividad dan lugar a la interpretación y la introspección.

En tercer lugar, acorde a las teorías subalternas, sus contrapartes positivistas parten de un conjunto de supuestos incuestionables sobre el ámbito internacional. Estos supuestos, aquí llamados fundacionalismos, atraviesan temas como la incuestionable condición de los individuos, la creencia en el método científico para llegar a la verdad, la inexistencia de una teoría del Estado y la razón como elemento emancipador.

El citado segundo gran debate de las Relaciones Internacionales acapara muchos de los fundacionalismos mencionados. De una parte, los realistas clásicos tradicionalistas como Morgenthau y Aron hacen hincapié en la naturaleza humana como variable explicativa de la anarquía del sistema internacional. Inspirados en las obras de Nicolás Maquiavelo y Thomas Hobbes, estos realistas retoman el pesimismo antropológico de ambos filósofos para transferirlo al Estado, al igual que el individuo, es una entidad egoísta, ambiciosa y agresiva. ${ }^{36}$ Esta identidad fijada del Estado/individuo permite que los análisis positivistas propios del Realismo faciliten la labor de concepción de comportamientos predecibles y de regularidades.

Los neorrealistas como Waltz juzgan como carente de cientificidad esta predisposición del hombre y los Estados a la anarquía y la guerra, trayendo a las Relaciones Internacionales los aportes de la revolución behaviorista. La aspiración a la unidad de las ciencias y la adopción del método científico como único camino a la explicación de las leyes sociales internacionales se mantuvo inclusive en la exploración de la síntesis neo-neo. ${ }^{37}$

Aunque las diferencias por el modo de demostración de las teorías podrían alejar a los Realistas clásicos y a los Neorrealistas, un factor que ambos comparten es la carencia de una teoría del Estado. De la humanización de este realizada por los clásicos a presupuestos compartidos como sus atribuciones de actor unitario y racional no hay un cuestionamiento con respecto a la constitución del Estado como producto social. Este carácter del Estado como un ente presocial es criticado por las teorías subalternas las cuales juzgan que la inexistencia de una teoría del Estado impide problematizar sus atribuciones e indagar sobre su preeminencia como actor internacional.

El tema de la razón como herramienta de la emancipación humana no es del todo atacado por las teorías subalternas. La idea del proyecto de la ilustración sobre el poder de la razón para liberar las mentes de los individuos es vista por teorías como el

\footnotetext{
35 Neufeld, op. cit, 1995, p. 53.

36 Ghazi A. R. Algosaibi, "The Theory of International Relations: Hans J. Morgenthau and His Critics", Background 8:4, febrero de 1965, 221-256.

37 Robert Jervis, "Realism, Neoliberalism, and Cooperation: Understanding the Debate”, International Security 24:1, verano de 1999, 42-63.
} 
Posestructuralismo como un fundacionalismo carente de problematización, pero para la Teoría crítica hay que desenvolver aún más esta discusión. Discusión que será ampliada más adelante en este artículo.

Todos estos funcionalismos tienen para las teorías subalternas su raíz en lo que las teorías subalternas han dado en llamar el "sentido común". Definido por Mark Rupert como una "amalgama de ideologías, de doctrinas científicas y de mitologías sociales" 38 este sentido común logra moldear el imaginario cotidiano de amplios sectores de la sociedad. Esta amalgama sin embargo no es algo natural o dado. Es producto de una perspectiva particular (occidental) desde la cual se establecen luchas de las fuerzas sociales por la preservación, resistencia o transformación de las relaciones de poder existentes. ${ }^{39} \mathrm{El}$ sentido común por lo tanto es un escenario de confrontación en vez de una categoría fija.

\section{El historicismo y el interpretivismo como ejes articuladores de las teorías subalternas.}

Como ha sido ya visto, las teorías subalternas hacen una apuesta por poner en evidencia 1as verdades inobjetables de las corrientes principales de las Relaciones Internacionales. Para llegar a ello, las teorías subalternas critican el determinismo histórico y el ahistoricismo de estas corrientes a la vez que realizaron una propuesta acerca de una nueva hermenéutica de la disciplina.

Por un lado, el afán positivista por aplicar la idea de leyes totalizantes permea su modo de observar la historia. Ver la historia desde la continuidad puede, a juicio de las teorías subalternas, conllevar a narrativas deterministas que justifican la configuración actual del sistema internacional como algo inevitable. Desde Tucídides y su visión cíclica de la historia en la narración de la guerra del Peloponeso, pasando por Maquiavelo ${ }^{40}$ y su determinismo histórico basado en la recurrencia de los acontecimientos y la invariable condición humana hasta Morgenthau ${ }^{41}$ y su extensiva alusión a acontecimientos históricos, las visiones realistas han hecho el mayor aporte al talante ahistoricista de las Relaciones Internacionales. La conexión arbitraria de eventos del pasado ha servido al paradigma realista para narrar el devenir de la historia humana y los efectos actuales como irremediables. Imposibles de ser modificados o rectificados hacia el futuro. De tal modo, la historia es contada desde una suerte de textos sagrados de una única manera y con una única conclusión.

La historia bajo una óptica totalizante no es un sesgo exclusivo de las corrientes principales de las Relaciones Internacionales. Las vertientes marxistas comparten una epistemología de la historia a la luz de leyes o estadios homogéneos cuyas relaciones de producción determinaron eras completas en todas las civilizaciones. El comunismo primitivo, el esclavismo, el feudalismo y el capitalismo se dieron de forma simultánea y uniforme durante siglos y hasta milenios. Sin excepciones y con escasas derivaciones. A

\footnotetext{
38 Mark Rupert, "Globalising common sense: A Marxian-Gramscian (re) vision of the politics of governance/resistence", Review of International Studies 29:1, 181-198.

39 Abrahamsen, op. cit, p. 112

40 Nicolás Maquiavelo, El príncipe, Madrid, Tecnos, 2000.

41 Hans Morgenthau y Kenneth Thompson, Politics among Nations, Nueva York, McGraw-Hill, 1985.
} 
pesar de que el marxismo estudia estas leyes históricas con el propósito de la transformación en lugar de la conservación, el halo de inevitabilidad se mantiene en este paradigma.

En las teorías subalternas, el determinismo histórico y la lectura totalizante de la historia dan paso al historicismo. Inspirado en Giambattista Vico, el historicismo piensa "en las instituciones humanas como algo hecho por la gente, no por gestos individuales de 'actores', sino como respuestas colectivas a problemáticas colectivamente percibidas y que producen ciertas prácticas. Instituciones y prácticas deben ser entendidas por lo tanto a través del cambio en los procesos mentales de sus creadores". ${ }^{42}$ En el Neogramscismo, el historicismo les ha servido a autores como Robert Cox para adelantar un programa de investigación que le permita hacer un examen en las transformaciones de las fuerzas productivas tanto en las formas de Estado como en los órdenes mundiales. ${ }^{43}$

Para los posestructuralistas como Richard Ashley, el historicismo debe ser el eje de la práctica de teorización. Para él, esta necesidad se desprende de dos premisas: i) la dependencia de la práctica sobre la estructura, ya que la significación y la prácticas del poder al momento de hacer la historia dependen de estructuras institucionalizadas, convenciones o preconcepciones que son tomadas como dadas y ii) esta dependencia de la estructura en la práctica parte de una estructura de verdades socialmente vívidas, la cual está siempre en proceso de ser impuesta en la historia y a través de las prácticas de los sujetos. ${ }^{44}$ No obstante, otros autores posestructuralistas como R.B.J. Walker hallan ciertas ambigüedades en el historicismo, particularmente en su confrontación con el Realismo clásico. Para él, los binomios universalismo/pluralismo, estructura/historia e identidad/diferencia son la base de esta ambigüedad. En especial, el último binomio puede resultar problemático puesto que el ponderar la diferencia sobre la identidad terminaría llevando a volver estos dos términos como mutuamente excluyentes. Una exclusión cuyos efectos podrían terminar por menoscabar virtudes de este realismo como su énfasis en el pluralismo, el cambio y la diferencia. 45

En términos metodológicos, la presencia del historicismo en las teorías subalternas es desarrollado usualmente por medio de la técnica de la genealogía. La genealogía se presenta como una alternativa de representación histórica que privilegia la resistencia sobre la significación y las conjeturas sobre las causas. La genealogía es un enfoque histórico orientado en los procesos y opuesto a la argumentación determinista de las corrientes principales de las Relaciones Internacionales que muestran etapas históricas como inevitables o adjudican a variables aisladas la respuesta a los cambios estructurales. Esta técnica ayuda a observar los cambios y continuidades en los discursos dominantes acerca

42 Traducción libre del autor. El texto original reza: "human institutions are made by people -not by the individual gestures of "actors" but by collective responses to a collectively perceived problematic that produce certain practices. Institutions and practices are therefore to be understood through the changing mental processes of their makers". Cox y Sinclair, op. cit, pp. 51-52.

43 Ídem.

${ }^{44}$ Ashley, op. cit, p. 273.

45 R.B.J. Walker, Inside/Outside. International Relations as Political Theory, Cambridge, Cambridge University Press, 2011, p. 123. 
de la conformación de las estructuras sociales desde narrativas en confrontación. Narrativas que plasman el modo en que los sujetos negocian, reproducen o cambian significados. ${ }^{46}$

El recurso a la genealogía entonces representa un desafío a dos de los más relevantes modos del ahistoricismo en los estudios internacionales: el cronofetichismo y el tempocentrismo. Entre ellos:

se dice que el primero denota 'sellar' el presente de tal manera que aparece como una entidad autónoma, natural, espontánea e inmutable. El último se refiere a la extrapolación de este presente 'naturalizado' hacia atrás en el tiempo de manera discontinua. Las rupturas y las diferencias entre épocas históricas y sistemas de Estados se suavizan y se oscurecen consecuentemente. ${ }^{47}$

El historicismo en general, y la técnica de la genealogía en particular, sugieren observar la historia como un ámbito de contingencias y de complejidad para alejarse de la prelación dada por las corrientes teóricas principales a la parsimonia teórica y a la visión totalizante de la historia. La prioridad será entonces explorar rupturas históricas y diferencias esenciales para identificar alternativas para la reorientación del orden internacional. 48

Por otro lado, las teorías subalternas, en su carácter interpretivista, realizan un aporte al entendimiento del sistema internacional al teorizar las instituciones que regulan el orden mundial. Para exponentes de la Teoría crítica como Marc Neufeld, la existencia social es un proceso dinámico de auto-interpretación y auto-definición de las colectividades humanas. Consecuentemente, las ciencias sociales son interpretativas en un doble sentido: i) en una "red de significado" que está comprendida por significados intersubjetivos que son producto de las auto-interpretaciones y las auto-definiciones colectivas de las comunidades humanas, las cuales no pueden ser asimiladas a la suma total de los significados subjetivos individuales $y$ ii) en una relación constitutiva y de no correspondencia entre "red de significado" y la práctica humana constitutiva, así la premisa naturalista de que las regularidades del mundo social son como las del mundo natural, independientes del tiempo y del espacio, debe ser rechazada. ${ }^{49}$

Para las teorías posestructuralistas, el interpretivismo también juega un papel crucial en el entendimiento de la configuración del orden internacional actual. Según David Campbell, el enfoque interpretivista se distingue por ver la teoría como práctica. En ese orden de ideas, la teoría de las Relaciones Internacionales llega a ser una instancia de un sitio de las prácticas culturales generalizadas que sirven para disciplinar la ambigüedad. ${ }^{50}$ Jim George pondera el perfil interpretivista del posestructuralismo al problematizar asuntos

\footnotetext{
${ }^{46}$ Audie Klotz y Cecelia Lynch, Strategies for Research in Constructivist International Relations, Nueva York y Londres, M.E. Sharpe, 2007, p. 31.

47 Andrew Bennett y Colin Elman, "Historical Methods", The Oxford Handbook of International Relations, Oxford, Oxford University Press, 2008, 518-538.

48 Íbid, p. 532.

49 Neufeld, op. cit, 1993, pp. 46-47.

50 David Campbell, Writing Security. United States Foreign Policy and the Politics of Identity, Minneapolis, University of Minnesota Press, 1998, p. 19.
} 
como: el legado de la modernidad occidental para el entendimiento contemporáneo del mundo real y del ser real, del sujeto moderno, de exclusión, el poder político y la vida de la periferia (la cuestión de la disenso y la resistencia) y la relación entre saber y poder, sobre todo del cómo respecto a las condiciones sociolingüísticas de la construcción de formas de saber dominante y de su disciplinamiento y representación de la vida contemporánea. ${ }^{51}$

En el plano metodológico, este interpretivismo encuentra en el análisis de discurso una técnica adecuada para su ejercicio de problematización del orden internacional y de las instituciones y subjetividades derivadas. Para los posestructuralistas, las ortodoxias de nuestros mundos políticos y sociales son recreadas mediante el proceso de escritura, en el estilo de los textos a través de los cuales son construidos los significados dominantes del mundo. ${ }^{52}$ Este análisis de discurso no se agota en la interpretación textual o subtextual, sino que se extiende hasta la intertextualidad. Este presupuesto tiene sentido pues los tomadores de decisión se sitúan en una esfera política y pública más amplia y sus representaciones deben ser, en consecuencia, diseñadas para articularse con las representaciones de individuos e instituciones. ${ }^{53}$

Sin embargo, la interpretación del discurso debe ir más allá del plano ideacional. La ontología discursiva del posestructuralismo estima que el discurso no debe equivalerse solamente a ideas. Para ellos, el discurso encierra igualmente elementos materiales. En su epistemología, el análisis de discurso en las Relaciones Internacionales implica la observación de la construcción de las identidades desde la perspectiva de la política exterior tanto para constituirla como para ser un producto de ella. Esta codeterminación entre discurso y política exterior es consecuente con el énfasis ontológico en el lenguaje y conlleva a enfocarse en el modo en que identidades y decisiones políticas están articuladas. ${ }^{54}$

Historicismo y interpretivismo reiteran entonces la relación intrínseca entre hechos y valores. La concepción de la historia como un proceso discontinuo avala el entendimiento del observador sobre la configuración de ordenes mundiales, prácticas, instituciones y subjetividades. Dicho entendimiento logra estar contextualizado desde unas relaciones de poder y de lenguaje específicas de un espacio y un tiempo determinados. La interpretación del estado actual de las cosas desde esta perspectiva subalterna facilita por tanto desvelar a la disciplina de las Relaciones Internacionales a través de la identificación y la intersubjetividad de los múltiples actores involucrados.

\section{Los debates al seno de las teorías subalternas.}

A pesar de haber fijado hasta este punto varios lineamientos en común de las teorías subalternas, el diálogo entre estas con el fin de pensar en una síntesis teórica está lejos de llegar a un consenso. La polémica por la razón y si verdaderamente todas ellas defienden una epistemología desde abajo dificultan una conciliación en el horizonte próximo.

\footnotetext{
51 Jim George, Discourses of Global Politics. A critical (Re) Introduction to International Relations, Boulder, Lynne Rienner, 1994, pp. 155-156.

52 Ashley, op. cit.

53 Lene Hansen, Security as Practice. Discourse analysisis and the Bosnian war, Londres y Nueva York, Routledge, 2006, p. 20.

54 Ídem.
} 
En primer lugar, la creencia en el alcance del proyecto de la ilustración y de la razón como medio para la emancipación humana están en el centro de la disquisición entre la teoría crítica y los posestructuralistas. En el núcleo de esta disquisición se halla una tensión entre la teoría de Jürgen Habermas y los posmodernos. Esta tensión se transporta al campo de las Relaciones Internacionales puesto que ambos son inspiradores de teorías como la Teoría crítica y el Posestructuralismo respectivamente. El posestructuralismo juzga al "nuevo racionalismo" de los 1980 y 1990 como una reencarnación del viejo racionalismo cuyas ilusiones y peligros continúan perpetuando una metanarración modernista. Para ellos, el sueño de la emancipación humana, concebido desde la ilustración, nunca logró cristalizarse, lo cual reduciría las ideas de Habermas a una variante de la metanarración modernita kantiana. ${ }^{55}$

Los autores de esta corriente profundizan sus cuestionamientos a la teoría crítica y a su compromiso con el supuesto poder emancipador del ideario de la ilustración. Para ellos, la ilusión utópica de la ilustración como medio para la obtención de la paz perpetua encubre el lado más oscuro de este planteamiento. La pretensión de llevar a cabo la ilustración sin con ello recurrir a un ejercicio directo de su voluntad que genere resistencia terminaría llevando al establecimiento de una hegemonía internacional. Por lo tanto, para desnudar este ideario, los posestructuralistas rescatan la obra de Michel Foucault y la noción de la política moderna como continuación de la guerra. Solo así, piensan ellos, se pueden entender los orígenes tecnoestratégicos de la capacidad de persuasión del discurso ilustrador. De ahí el llamado a romper con el legado de la ilustración y de la Revolución francesa. ${ }^{56}$

Precisamente, la Revolución francesa como culmen del proyecto ilustrador encaja dos eventos que atestiguan las contrariedades de la ilustración. Ella exalta por igual los altos valores de los derechos del hombre como la coexistencia de él con el terror. Lo ocurrido después de ella sin embargo es la emergencia de una sociedad más militar y represiva que la del ancien regime, cosa que condujo a la aparición de una sociedad disciplinaria basada en la vigilancia. ${ }^{57}$

La fe depositada en la ilustración de Habermas y los exponentes de la teoría crítica trae implícita para los posestructuralistas la sobrevaloración de la razón como factor emancipatorio. Esta preeminencia de la razón es acusada por estos últimos de ser un fundacionalismo de la teoría. Un fundacionalismo que eventualmente conduciría a un universalismo impositivo. La insistencia en el poder de la razón es percibida por los escépticos de la teoría crítica como un afán por retrotraer un conocimiento que ha sido sujeto continuamente de valoraciones críticas. Incluso se llega al punto de asimilar a la teoría crítica con lo que Robert Cox denomina las teorías de resolución de problemas. 58

En el sentido opuesto, desde la Teoría crítica se reivindica a la razón para responder de dos modos a las críticas posestructuralistas. De un lado, se toma la crítica a la razón como fundamento de la emancipación y se denuncia una asociación juzgada no procedente. Para

\footnotetext{
55 George, op. cit. P. 187.

56 Der Derian, op. cit, p. 303.

57 Ídem.

58 George, op. cit, p. 187.
} 
los teóricos críticos, los posestructuralistas equiparan razón con racionalismo instrumental. Esta falsa asociación se encuadra en lo que lo teóricos críticos han llamado "la irracionalidad de Foucault". Un menosprecio de las ideas de Kant y de Weber que los orienta a realizar esta asociación. Del otro lado, los teóricos críticos resaltan otro problema de los posestructuralistas al atribuirles una postura que se limita a una representación neutral, y que no toma partido, de voces en enfrentamiento, impidiéndoles tomar partido por agendas políticas y sociales que busquen reconstruir la política mundial. Es por ello que, los posestructuralistas "deberían aspirar a reconciliar algunas formas de universalismo con el pluralismo y la diferencia en vez de rechazar el universalismo". ${ }^{59}$

En segundo lugar, la pretensión de privilegiar una epistemología "desde abajo" de las Relaciones Internacionales no convoca de la misma manera las distintas vertientes de las teorías subalternas. Centrar el objeto de análisis en "los oprimidos del mundo" no es una tarea ausente de polémica entre las teorías subalternas. Entre ellas, por un lado, se encuentran las que privilegian un análisis más cercano al marxismo y por el otro lado, las que intentan ir más allá. En la primera postura, se ubican el Neogramscismo, la Teoría crítica y el Feminismo materialista. En la segunda postura, el Posestructuralismo, el Poscolonialismo y sus sendas vertientes feministas.

Las teorías correspondientes a la primera postura parten de la idea de la división social en clases para colocarse en la perspectiva de los subordinados o dominados. En el Neogramscismo, la dualidad entre clase capitalista/clase proletaria da lugar a una relación dialéctica que comporta factores ideológicos en esa interacción. El paso a la contraposición entre el bloque histórico y las clases subalternas hace de las segundas el foco del análisis neogramsciano para diseccionar el potencial de la contrahegemonía. ${ }^{60}$

En la teoría crítica hay una mayor afinidad con el precepto marxista del proletariado. Particularmente, autores como Andrew Linklater restablecen el planteamiento marxista del proletariado internacional como sujeto del cambio social y de la visibilización y dignificación de los derechos y las condiciones materiales de existencia de los dominados. ${ }^{6}$ En el feminismo materialista, la idea de división social del trabajo es reformada por la de división sexual del trabajo para hallar relaciones de dominación incluso desde lo que Marx nombró el comunismo primitivo. La instauración de la dicotomía público/privado para asignar roles a partir del género y la constitución de un Estado falocéntrico reforzaron estas relaciones sexuales de producción.

Respecto a la segunda postura, los posestructuralistas y poscolonialialistas reconocen el aporte marxista, pero disciernen en tratar a los subordinados desde categorías homogéneas. Los primeros trasladan al plano microfísico las relaciones de poder que crean corporeidades y subjetividades. Los segundos anteponen a la visión etnocentrista occidental una desprendida del "Sur". En ambos casos, se cuestionan las categorías de "dominados" y "tercer mundo" como entidades monolíticas, las cuales dificultan observar las prácticas de dominación en todo lugar donde ello ocurra. Dentro de estas vertientes, las feministas

\footnotetext{
59 Neufeld, op. cit, 2002, p. 115.

60 Cox y Sinclair, op. cit.

61 Andrew Linklater, "The Problem of Community in International Relations", Alternatives: Global, Local, Political 15:2, verano de 1990, 135-153.
} 
rechazan centrar su análisis en la clasificación integral de "mujeres del tercer mundo" o "mujeres disciplinadas" puesto que se alejaría del entendimiento de las prácticas de opresión específicas para cada caso. El estudio de las mujeres entonces se hace intersectorial y prioriza las historias particulares sobre sus luchas individuales. ${ }^{62}$ En consecuencia, la segunda postura desciende aún más que la primera en su epistemología "desde abajo".

\section{La normatividad de las teorías subalternas: el camino a la emancipación}

A pesar de que existen diferencias significativas entre las teorías subalternas en su ontología y especialmente en su epistemología, lo cual tendrá una repercusión sobre su normatividad, también deben contemplarse unas líneas de prescripciones que las acercan. Estas líneas guardan concordancia con i) lo explícito de su normatividad, ii) la amplitud de la comunidad y de los valores que a este le pertenecen y iii) su intención transformadora.

\section{Las teorías subalternas como teorías con una normatividad explícita.}

Uno de los principales puntos en común de las teorías subalternas es el hecho de contar con una normatividad explícita y problematizada, pero divergente en algunos aspectos. Neogramscianos y teóricos críticos promueven como objetivo de sus teorías la transformación de sujetos dominados a sujetos autónomos. Para los primeros, la combinación entre conocimiento, conciencia y acción humanos haría factible el posicionamiento de una agenda poshegemónica que se ofrezca como una alternativa de transformación a la producción histórica del capitalismo global.63 Para los segundos, el pilar determinante del cambio social está en la conciencia. El paso del individuo dominado al emancipado se halla en el paso de la inconciencia a la conciencia. Así, los enfoques interpretativos de las Relaciones Internacionales sirven para reconocer a este campo como algo que no está "dado", sino que es "elaborado" y por lo cual es capaz de ser "reelaborado". Este enfoque interpretativo permite teorizar un cambio tanto dentro de las instituciones que regulan el orden mundial como del orden mundial mismo. ${ }^{64}$

El anhelo utopista de la Teoría crítica es compartido de alguna manera por el Poscolonialismo. Los teóricos críticos perfilan la teoría hacia la instauración de un orden racional, justo y democrático en la escala mundial. Los poscolonialistas, si bien critican el desarrollo del orden internacional con la acción de tipos de violencia específicos, no declinan en la idea de un orden global diferente. La influencia de varios pensadores eclesiásticos, de la moral y la ética fue de utilidad para que esta vertiente defendiera la idea de "hermandad".

\footnotetext{
62 Abrahamsen, op. cit. P. 111.

63 Stephen Gill, Gramsci, Historical Materialism and International Relations, Cambridge, Cambridge University Press, 1993, p. 17.

64 Neufeld, op. cit, 2002, p. 93.
} 
Una hermandad no obstante expresada de manera diferente a la que promovieron desde Europa. 65

En el Posestructuralismo y algunos feminismos la normatividad está más relacionada con la resistencia. El posestructuralismo se proyecta a sí mismo como el pensamiento de la disidencia en las Relaciones Internacionales. Su antifundacionalismo característico pone en cuestionamiento los regímenes de verdad y el saber/poder puesto en escena en los discursos de los dirigentes mundiales para deconstruirlos con el fin de desafiarlos y subvertirlos. Feministas como las posestructuralistas hacen una labor similar al deconstruir los discursos que crean las categorías de género y crean un universo de sentido para los sujetos involucrados. ${ }^{66}$

\section{¿Los valores de quién?}

Una segunda línea de confluencia normativa entre las teorías subalternas es la relativa a cuál es la comunidad de valores promovidos desde estas perspectivas. En todos los casos, los valores son los pertenecientes a aquellos que son marginados por las narraciones dominantes de las Relaciones Internacionales. El Neogramscismo acusa directamente a las corrientes principales de la disciplina (Realismo, Neorrealismo y Liberalismo) como instrumentos de opresión para la mayor parte de la humanidad, creando una preocupación de esta teoría por los explotados y marginados. De ahí la importancia de ponderar las prácticas contrahegemónicas en busca de constituir un nuevo bloque histórico que sea más representativo de los intereses del conjunto de la humanidad.

En un amplio espectro del feminismo, así como en el Poscolonialismo, se comparte el rechazo a la lectura etnocéntrica que hace el Realismo del acontecer internacional. Para ellos, la teorización los obliga a inquirir por una gama muy amplia de autonomías localmente entendidas con el deber de "reconfigurar el mundo y 'rehusar' contemplar lo bueno y lo justo desde solamente un lado". ${ }^{67}$

Igualmente, los posestructuralistas se ponen del lado de aquellos que son silenciados o marginados por los relatos ortodoxos y cuyas subjetividades son descartadas por la visión de alteridad que es representada por esos relatos. Según los teóricos críticos, el discurso puede también reflejar una ética que favorezca el verdadero diálogo sobre el ejercicio mundial del poder. Un discurso del cual participen en igualdad de condiciones los movimientos sociales, los actores no estatales, los ciudadanos mundiales, los Estados y las organizaciones internacionales. La finalidad de este ejercicio dialógico es fomentar en la comunidad entera una actitud cosmopolita en la cual todo ser humano goce de los mismos derecho y obligaciones sin importar la jurisdicción territorial en la que se encuentre. De tal modo, el proyecto emancipador pasará por la ampliación de las fronteras morales de toda la comunidad política.

\footnotetext{
65 Siba N. Grovogui, “Postcolonialism”, Tim Dunne, Milja Kurki y Steve Smith (eds.), International Relations Theories. Discipline and Diversity, Oxford, Oxford Univseristy Press, 2010, 247-265.

66 J. Ann Tickner, Gendering Worls Politics. Issues and Approaches in the Post-Cold War Era, Nueva York, Columbia University Press, 2001.

67 Sylvester, op. cit.
} 


\section{Las teorías subalternas como teorías transformadoras.}

A pesar de discordar en las maneras de proceder, las teorías subalternas persiguen como fin último la transformación del orden social internacional en favor de los excluidos y marginados. En el entendimiento de la estructura que debe ser subvertida, se hallarán los matices fundamentales para el diálogo teórico.

En los neogramscianos, la transformación del opresivo sistema actual se desprende de la noción de contrahegemonía. La contrahegemonía refiere a la comprensión de los órdenes mundiales como producto de las relaciones sociales. Teniendo en mente esta comprensión, el cambio estructural de ese orden mundial debe ser rastreado justamente en esas relaciones sociales y en los órdenes políticos nacionales que se corresponden a ellas. Por lo tanto, para Robert Cox "[...] la tarea de cambiar los órdenes mundiales comienza por la ardua labor de construir de nuevos bloques históricos al interior de las fronteras nacionales". ${ }^{68}$ Los neogramscianos proponen entonces una hegemonía "de abajo hacia arriba", pero enquistada en las prácticas sociales locales.

En el sentido opuesto, los teóricos críticos toman partido por una transformación "de arriba hacia abajo" haciendo hincapié en el universalismo. Para ellos, la disciplina de las Relaciones Internacionales le ha fallado al "proyecto aristotélico" al evitar ubicar el asunto de la emancipación humana en el centro de las preocupaciones teóricas. Una emancipación distinguida por tres elementos cruciales: el reflexivismo teórico, la atención en el papel jugado por la creatividad de la conciencia humana y la preocupación por el criticismo social apoyado en una actividad política práctica guiada hacia el cambio social radical. ${ }^{69} \mathrm{Al}$ combinar la puesta en marcha de estos tres elementos, los teóricos críticos no solamente ponen de relieve una disyuntiva a la preeminencia del positivismo, sino que también proponen estrategias de actuación encaminadas a elevar el perfil de la protección de los derechos humanos, priorizar la acción colectiva para mejorar las condiciones materiales de los miembros más pobres de la sociedad y rescatar el legado de la ética global y la responsabilidad por la naturaleza. ${ }^{70}$

Ante estas epistemologías de actuación unidireccional, las otras tres teorías subalternas han optado por conservar la tensión entre universalismo y particularismo. Aunque el Posestructuralismo y ciertos feminismos evalúan la producción de subjetividades y por ello intentan rescatar las microhistorias de estos sujetos, la centralidad de sus análisis en las estructuras discursivas de los expertos y conocedores y en la necesidad de subvertir esas estructuras (de dominación) mantienen la atención también sobre el carácter de "arriba hacia abajo" del poder. ${ }^{71}$ Este doble flujo del poder es resumido por la feminista Cynthia Enloe cuando afirma "lo personal no es solo político, sino internacional e internacional en lo personal. Aceptar que lo personal es internacional multiplica a los

68 Cox y Sinclair, op. cit. p. 140.

69 Neufeld, op. cit, pp. 20-21.

70 Linklater, op. cit, pp. 150-151.

71 Tickner, op. cit. 
espectadores, especialmente agrega a las mujeres a la audiencia, pero no transforma lo que está pasando en el escenario".72

A pesar de que el Poscolonialismo comparte este enfoque de doble flujo entre el universalismo y el particularismo, su deber ser parece darle mayor importancia al segundo. Su foco en los símbolos y las prácticas occidentales y su precisión de observar la relación de dominación Norte-Sur más allá de la unidireccionalidad contrastan con un agenciamiento de resistencia a través del concepto que ellos denominan "hibridación". La hibridación es un sitio potencial de resistencia y subversión que procura romper con la distinción entre el sí y la alteridad. Este concepto apela a la adaptación y transformación creativa de esos símbolos y prácticas occidentales para dar voz y visibilidad a los marginados. ${ }^{73}$

\section{Conclusiones}

Este artículo se orientó por visualizar la posibilidad de mundos alternativos al actual a partir del análisis de la integridad teórica interna de las teorías subalternas de las Relaciones Internacionales. La tarea que aquí se emprendió fue más allá del seguimiento de un camino lineal. A pesar de coincidir en la visualización de una transformación social e internacional, las denominadas teorías subalternas se encuentran lejos de encontrar consenso sobre la manera en que este cometido debe conseguirse.

Uno de los temas remarcables al momento de realizar este ejercicio radica en que la categoría "subalterna", asignada para las teorías estudiadas, tiene raseros comunes como el rechazo al positivismo atribuible a las corrientes principales de las Relaciones Internacionales y a la denuncia de las estructuras ideacionales de dominación. Por un lado, para estas teorías la racionalidad positivista apunta a naturalizar visiones particulares y etnocéntricas del sistema internacional, a someter a las ciencias sociales a las reglas metodológicas de las ciencias naturales y a justificar el estatus quo reinante mediante la prescripción de asepsia valorativa como directriz del observador. Por el otro lado, las teorías subalternas encuentran en el historicismo y en el interpretivismo elementos de comunión para conjurar el determinismo histórico de las teorías positivistas y para entender los procesos que han llevado al estado actual de las cosas.

Sin embargo, estos raseros no alcanzan a ocultar las diferencias sustantivas en la teorización y prescripción de los acontecimientos internacionales. Sus posturas con respecto al potencial emancipador de la realidad y del foco seleccionado como el "abajo" de las Relaciones Internacionales inhibe a los pensadores de estas perspectivas en contra de crear frentes comunes de entendimiento para contrarrestar los efectos de las interpretaciones instrumentales del mundo circundante. Además, la disputa por la razón en el modo emancipador en el cual fue preconizada por el proyecto de la ilustración se vislumbra como

\footnotetext{
72 Traducción libre del autor. El texto original reza: “The personal is not just political but international and the international in personal "accepting that the personal is international multiplies the spectators, it especially adds women to the audience, but it fails to transform what is going on the stage". Cynthia Enloe, "Margins, silences, and bottom rungs: how to overcome the underestimation of power in the study of international relations", Steve Smith, Ken Booth y Marysia Zalewski (eds.), International theory: Positivism \& beyond, Cambridge University Press, Cambridge, 1996, 186-202.

73 Abrahamsen, op. cit, pp. 118-119.
} 
un punto muerto entre teóricos subalternos. La diferencia entre la defensa de una moral universal y la posibilidad de que esa moral sea implementada como un discurso totalizante da la impresión de convertirse en un callejón sin salida a la construcción colectiva de un frente de teorización (y de acción) desde lo subalterno.

Si bien las discrepancias en las visiones subalternas dan la impresión de ser insuperables, ello no puede ocultar las posibilidades de acción ofrecidas por estas teorías, las cuales son variadas y tienen la virtud de ofrecer diversas perspectivas del orden internacional construido hasta ahora. Su pretensión por llevar a la praxis la esperanza y la reivindicación de las aspiraciones prometidas hace siglos no deben ser omitidas por una disciplina que se precia de ser holística. Su voluntad de romper con el objetivismo y el falso naturalismo para reconocer el agenciamiento humano en el mundo exterior que ha sido producido o construido socialmente. Su valentía por dar voz y protagonismo a los silenciados y a los marginados por el sistema para reivindicar sus narraciones, pero sobre todo su impronta transformadora que invita a entrever otras posibilidades de relacionarse entre humanos son razones poderosas para invitar a no permitir el desfallecimiento de estos enfoques. Empero, no deja de causar temor que, al caer en el relativismo de la exaltación de las microhistorias y el rechazo a toda forma de conocimiento previo pueda contraer consecuencias fraccionadoras para un proyecto intelectual prometedor. Un proyecto cuyas vertientes están en mora de pasar del entendimiento del mundo a la concepción de estrategias de deconstrucción de las estructuras ideacionales de dominación. El riesgo de sobrepensar la acción colectiva puede terminar sepultando las alternativas a un mundo que no da espera. Ante esta última reflexión solo queda por preguntarse ¿Cómo articular lo deseable con lo viable?

\section{Bibliografía}

Rita Abrahamsen, "Postcolonialism", Martin Griffiths (ed.), International Relations Theory for the Twenty-First Centruy. An Introduction, Nueva York y Londres, Routlegde, 2007, 111-122.

Ghazi A. R. Algosaibi, “The Theory of International Relations: Hans J. Morgenthau and His Critics", Background 8:4, febrero de 1965, 221-256.

David Campbell, Writing Security. United States Foreign Policy and the Politics of Identity, Minneapolis, University of Minnesota Press, 1998.

Robert Cox y Timothy Sinclair, Approaches to World Order, Cambridge, Cambridge University Press, 1996.

James Der Derian, “The (S)pace of International Relations: Simulation, Surveillance and Speed", International Studies Quaterly 34:3, septiembre de 1990, 295-310.

Stephen Gill, Gramsci, Historical Materialism and International Relations, Cambridge, Cambridge University Press, 1993.

Robert Gilpin, War and change in world politics, Cambridge, Cambridge University Press, 1981.

Lene Hansen, Security as Practice. Discourse analysisis and the Bosnian war, Londres y Nueva York, Routledge, 2006. 
Martin Hollis y Steve Smith, Explaining and Understanding International Relations, Oxford, Clarendon Press, 1990.

Robert Jervis, "Realism, Neoliberalism, and Cooperation: Understanding the Debate", International Security 24:1, verano de 1999, 42-63.

Robert Keohane, Neorealism and Its Critics, Nueva York, Columbia University Press, 1986.

Robert Keohane, "International Institutions: Two Approaches", International Studies Quaterly 32:4, diciembre 1988, 379-396.

Audie Klotz y Cecelia Lynch, Strategies for Research in Constructivist International Relations, Nueva York y Londres, M.E. Sharpe, 2007.

Yosef Lapid, "The Third Debate: On the Prospects of International Theory in a PostPositivist Era", International Studies Quaterly 33:3, septiembre 1989, 235-254.

Andrew Linklater, "The Problem of Community in International Relations", Alternatives: Global, Local, Political 15:2, verano de 1990, 135-153.

Nicolás Maquiavelo, El príncipe, Madrid, Tecnos, 2000.

Hans Morgenthau y Kenneth Thompson, Politics among Nations, Nueva York, McGrawHill, 1985.

Marc Neufeld, "Interpretation and the 'Science' od International Relations", Review of International Studies 19:1, enero de 1993, 39-61.

Mark Neufeld, The restructuring of International Relations theory, Cambridge, Cambridge University press, 2002.

Mark Rupert, "Globalising common sense: A Marxian-Gramscian (re) vision of the politics of governance/resistence", Review of International Studies 29:1, 181-198.

Christine Sylvester, "The constributions of feminist theory to international relations", Steve Smith, Ken Booth y Marysia Zalewski (eds.), International theory: Positivism \& beyond, Cambridge University Press, Cambridge, 1996, 254-278.

J. Ann Tickner, Gendering Worls Politics. Issues and Approaches in the Post-Cold War Era, Nueva York, Columbia University Press, 2001.

Keith Tooper, "The Theory of International Politics? An Analysis of Neorealist Theory", Human Studies 21: 2, abril de 1998, 157-186.

R.B.J. Walker, Inside/Outside. International Relations as Political Theory, Cambridge, Cambridge University Press, 2011.

Kenneth Waltz, Theory of International Politics, Nueva York, Columbia University Press, 1979.

\section{Capítulos de libro}

Andrew Bennett y Colin Elman, "Historical Methods", The Oxford Handbook of International Relations, Oxford, Oxford University Press, 2008.

Cynthia Enloe, "Margins, silences, and bottom rungs: how to overcome the underestimation of power in the study of international relations", Steve Smith, Ken Booth y Marysia Zalewski (eds.), International theory: Positivism \& beyond, Cambridge University Press, Cambridge, 1996, 186-202.

Siba N. Grovogui, "Postcolonialism", Tim Dunne, Milja Kurki y Steve Smith (eds.), International Relations Theories. Discipline and Diversity, Oxford, Oxford Univseristy Press, 2010, 247-265. 
Alex Macleod, "Émergence d'un paradigme hégémonique", Alex Macleod y Dan O'Meara, Théories des relations internationales : contestations et résistances, Athéna éditions, Outremont, 2010, 19-35.

Mark Neufeld, "Reflexivism", Claire Turenne Sjölander y Wayne S. Cox (dirs,), Beyond Positivism : Critical International Relations Theory, Boulder, Lynne Rienner Publications, 1994. 13-45.

Steve Smith, "Six Wishes for a More Relevant Discipline of International Relations", Christian Reus-Smit y Duncan Snidal (eds.), The Oxford Handbook of International Relations, Oxford University Press, Oxford, 2008, 725-732.

Ole Waever, "The rise and fall of the inter-paradigm debate", Steve Smith, Ken Booth y Marysia Zalewski (eds.), International theory: Positivism \& beyond, Cambridge University Press, Cambridge, 1996, 149-185. 